\title{
Teams and Cardiac Surgery
}

\author{
Jan Maarten Schraagen \\ TNO Human Factors \\ Jan_maarten.schraagen@tno.nl \\ Paul Barach \\ New South Wales Injury Risk Management Center \\ p.barach@unsw.edu.au
}

\author{
Josine van de Ven \\ TNO Human Factors \\ Josine.vandeven@tno.nl \\ Meike Smit \\ TNO Quality of Life \\ Meike.smit@tno.nl
}

\begin{abstract}
Motivation - Our study is designed to identify human factors that are a threat to the safety of children with heart disease. Research approach - After an initial observation period, we will apply a major safety intervention. We will then re-measure the occurrence and types of human factors in the operating room, and the incidence of adverse events, near misses and hospital death, to evaluate if there was a significant post-intervention reduction. Findings/design - We focus on challenges encountered during the training of the observers. Research Limitations - Because of the complexity of the OR, observations are necessarily subjective. Originality/Value - This work is original because of the systematic evaluation of a safety intevention and the training protocol for the observers. Take Away Message - Systematic and periodic assessment of observers is required when teamwork is observed in complex, dynamic settings.
\end{abstract}

Keywords

Medical errors, patient safety, medical teams.

\section{INTRODUCTION}

Congenital heart surgical procedures are among the most complex pediatric interventions, encompassing anatomic diversity, hemodynamic vulnerability, and the need of a highly-skilled, multi specialty team. Increasing survival among children born with even the most complex cardiac defects has been achieved over the past three decades (Boneva, Botto, Moore, Yang, Correa, \& Erickson, 2001). Cardiac surgery shares many properties with high technology systems in which performance and outcomes depend on complex individual, technical, and organizational factors and their interactions. It depends upon a sophisticated structure where coordinated efforts of multiple individuals working as a team and high levels of cognitive and technical performance are necessary (de Leval, 1997).

Similarly to the surgical field, accidents in the air and railways industries are often found to be attributed to human error (Reason, 1990). While a particular human action or omission may be the immediate cause of an incident, closer analysis usually reveals a series of events and departures from safe practice, potentially influenced by the working environment and the wider organizational context. Understanding the characteristics of a safe and highly performing system therefore requires research on a number of interrelated topics. These include the specific context, the acquisition and maintenance of individual skills, the role of technology and the impact of working conditions on team performance. Safety in these organizations ultimately is understood as a characteristic of the system -the sum of all its parts and their interactions (Weick \& Quinn, 1999).

Research has shown that errors in the operating room occur within and between teams (Eichhorn, 1989; Keenan \& Boyan, 1985). Quite often, errors result from a breakdown in coordination and communication between the operating room sub-teams (Helmreich, 2000). For example, the scrub nurse and the surgeon failing to synchronize their actions so that blood is not sucked away from the surgical field quickly enough, obscuring the damaged blood vessel; or the anesthesiologists acting unilaterally because he has lost pace with the progress of the surgical team. Surgical errors have been identified as wrong-sided or wrong site surgical error in renal, orthopedic and thoracic cases (Cowell, 1998; Dambrosia \& Kilpatrick, 2002; Shapiro, Croskerry, \& Fisher, 2002) Malpositioning of surgical instruments during laparascopic cholecystectomy are known to lead to major vascular injury (Lemmo, Marocco-Trischitta, Manni, \& Snider, 2002), and misdiagnosis of unexpected complications (Macarthur, Nixon, \& Aitkin, 1998; Rao, Badani, Jamieson, \& Schildhauer, 1996). Errors made by operating room theatre scrub nurses include handing the wrong instruments to surgeons, and poor sterilization of surgical equipment (Wolfson, Seeger, Kadell, \& Eckart, 2000). Finally, the errors that other team members commit such as operating room orderlies and perfusionists during openheart surgery have been documented (Ducat, Merry, \& Webster, 2000; Jenkins, Morris, \& Simpson, 1997; Mejak, Stammers, Rauch, Vang, \& Viessman, 2000). Unclear instructions from the attending surgeon and anesthesiologist to their assistants (or vice versa) can also result in errors (e.g. failure to administer heparin before connecting the patient to the heart lung bypass machine). Faulty communication and follow through in the surgical intensive care unit have been 
identified as critical in the lack of error prevention and mitigation (Donchin, Gopher, Olin, Badihi, Biesky, Sprung, Pizov, 1995). Preoperative planning failures may also have a negative effect on performance in the operating room, for example poor quality or unavailable test results or patient notes, cross matching errors in the blood bank (Bonini, Plebani, Ceriotti, \& Rubboli, 2002; Williamson, Lowe, Love, Cohen, Soldan, McClelland, Skacel, 1999), failure to record known drug allergies or other key patient details (Cantrill, Cottrell, 1997). Another major source of error is the hand-off of responsibility for a patient by one health professional to another. The provision of health care services is a 24-hour a day, 7-day a week process, with shift work, interchange between multiple disciplines across culture and authority gradients, and management of a vast volume of shared critical information.

In similar complex environments, referred also as complex socio-technical systems, human factors research has been a major contributor to safety and reliability enhancement during the past 2 decades (de Leval, Carthey, Wright, Farewell, $\&$ Reason, 2000). The importance of human factors and systems research in improving outcomes for pediatric cardiac surgery has been highlighted in the Bristol Royal Infirmary and the Manitoba Inquiries (Murphy, 2001).

In the United Kingdom, Marc de Leval and collaborators used the model of organizational accident causation developed by Reason et al. (Reason, 1990) to develop a methodology with which to study performance in the neonatal arterial switch operation (ASO). Reason's theory distinguishes between active failures and latent conditions. Active failures are errors and violations that are committed by people at the service delivery end of the system (e.g. pilots, control room operators, financial traders, a ship's crew, and, in cardiac surgery, the operating room team). Active failures by these people have an immediate impact on safety. Latent conditions result from poor decisions made by the higher management in an organization, for example by regulators, governments, designers, and manufacturers. Latent conditions and active failures lead to windows of opportunity in a system's defenses. When these windows of opportunity are aligned across several levels of a system, an accident trajectory is created (Carthey, de Leval, \& Reason, 2001; Reason, 1990).

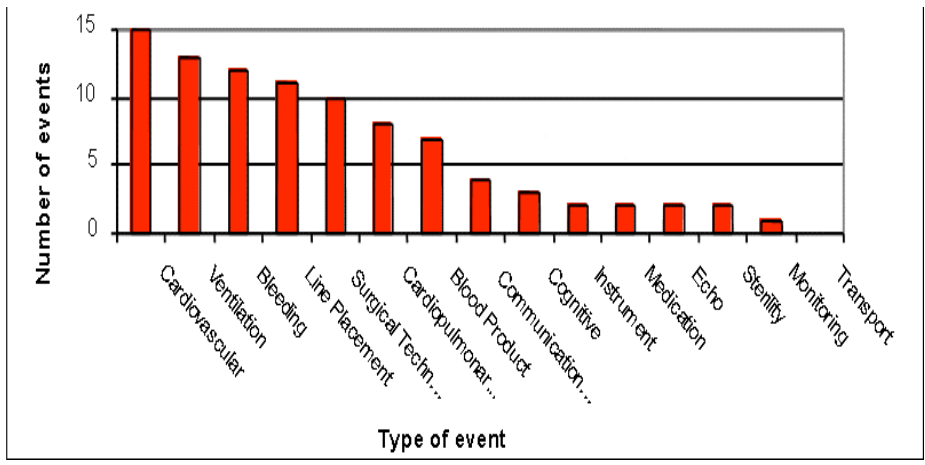

Figure 1. Distribution of Major Adverse Events

\section{PREVIOUS OBSERVATION STUDIES}

Earlier observation studies involved observing 102 cases of complex pediatric cardiac surgery (Barach, Johnson, Ahmad, Galvan, Bognar, Duncan, Starr, \& Bacha, 2008; Christian, Gustafson, Roth, Sheridan, Gandhi, Dwyer, \& Zinner, 2006; Galvan, Bacha, Mohr, \& Barach, 2005). They found that an average of 1.2 (range 0-6) major adverse events occurred per case. The most common type of major adverse events was cardiovascular (See Figure 1), and most occurred during the surgery/post-bypass surgical period. Cognitive compensation was the most common compensation mechanism for major adverse events (Figure 2). In addition, an average of 15.3 minor adverse events occurred per case. Minor adverse events occurred frequently during the surgery/bypass surgical period, and were related to communication and coordination failures. Case complexity $(\mathrm{p}<0.01)$ and surgery duration $(\mathrm{p}<0.05)$ were both significant predictors of major adverse events. In turn, a higher number of major adverse events per case correlated with post-operative death $(\mathrm{p}<0.01)$. We concluded that adverse events occurred routinely during pediatric cardiac surgery, and were mostly compensated. Case complexity was a significant predictor of major adverse events. The number of major adverse events per case correlated with clinical outcomes. Having thus established baseline data on human factors for pediatric cardiac surgery, we now aim to establish a rigorous, objective evidence-based approach to quality improvement, by doing a prospective pre-post intervention study (Mittman, 2004). 


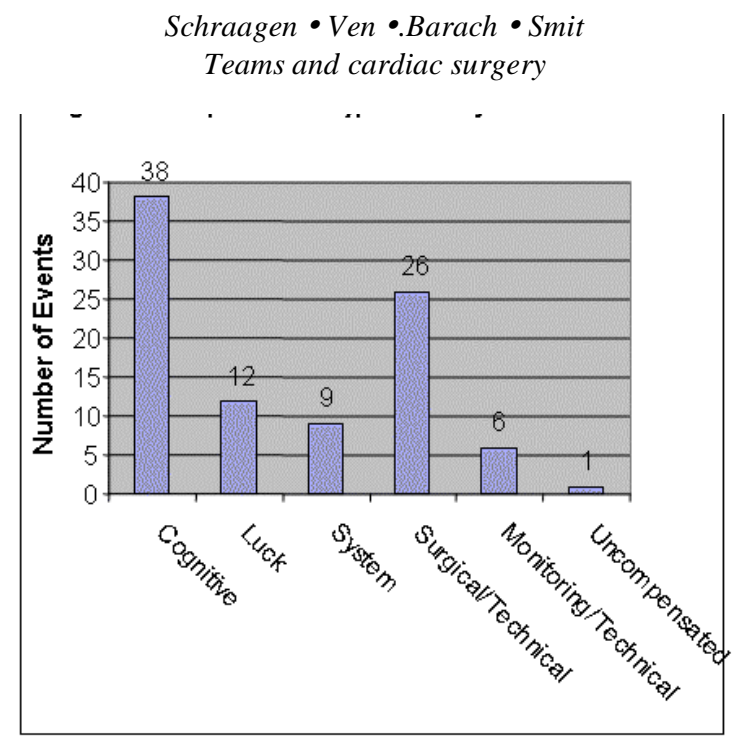

Figure 2. Compensation Types of Major Adverse Events

\section{CURRENT STUDY}

The purpose of this study was to evaluate the relationship between intraoperative adverse events and human factors within surgical teams in charge of pediatric cardiac surgery at the Utrecht Medical Center Children's Hospital. First, we identified a variety of human factors and evaluated the incidence of adverse events during pediatric cardiac surgery. Subsequently we performed a very detailed briefing of our findings (including a written summary to each team member) to the OR team. We endeavored to increase the team awareness of common adverse events during pediatric cardiac surgery, their compensation mechanisms, and the role of human factors. Finally, we re-evaluated the incidence of those adverse events to explore a possible reduction. Interactions of those with undesirable outcomes (a near-miss, permanent injury or death) were explored. We also identified the human-factor elements that lead to positive medical outcomes, that is, the counteractions taken by individuals or the team to neutralize an adverse event before it produces a undesirable outcome. With the final results of the study, we aim to provide important data to the pediatric cardiac surgical community.

\section{RESEARCH DESIGN}

\section{Terminology}

- Adverse events: unintended injuries to patients caused by healthcare rather than disease (Kohn, Corrigan, \& Donaldson, 2000).

a. Minor events: Failures that disrupt the "surgical flow" of the procedure but which, in isolation, were not expected to have serious consequences for the safety of the patient.

b. Major event: Failure that is likely to have serious consequences for the safety of the patient.

\section{Data Sources and Study Subjects}

The operating room will be considered a "microsystem" - a small, well defined front-line unit providing care for a specific patient population. The process of care will be divided into 7 surgical periods based on extensive observations (Galvan, Bacha, Mohr, \& Barach, 2005) and an iterative process: (1) Pre-op/Transport to OR; (2) Anesthesia induction; (3) Surgery pre-bypass; (4) Surgery bypass/repair; (5) Surgery Post-Bypass; (6) Transport to ICU; (7) Handoff to ICU team. Thus, observations will begin when transporting the patient to the operating room and end after hand-off to the intensive care unit.

\section{Training and assessment of observers}

Training for the two observers included in-depth directed study of cardiac surgery theory and literature, watching videotaped pediatric cardiac surgery procedures, and detailed discussions of ethnographic observational methods. The observers were encouraged to ask questions, and were informally tested by pediatric cardiac surgeons, cardiac anesthesiologists, cardiac perfusionists and OR nurses to ascertain the observer's knowledge and understanding of procedures and operating room dynamics and culture. Observers observed at least 10 live cases prior to collecting data. Prior to collecting data, observers had to pass an exam. The exam consisted of watching a 2 hour fragment of a videotaped operation, and scoring this fragment in real-time on a second-by-second basis. Interrater reliability was 
assessed by calculating the number of events scored by both observers and determining whether or not observers rated these events identically as far as teamwork was concerned. Interrater reliability was $91 \%$ at the level of the four main teamwork categories and $84 \%$ at the level of the 14 detailed subcategories. Acquisitition of relevant domain knowledge by the observers was assessed by an experienced anesthesist, in the following ways. First, the anesthesist interviewed the observers after the exam to check whether they had understood the procedure and whether they had noted the nonroutine events involved. Second, the anesthesist developed a set of 23 statements that could be either right or wrong. The set of statements was adminstered during a second exam midway through the first observation period. These statements were geared at the level of an experienced anesthesist. Taking into account chance levels, observers scored a ' 5 ' on a scale from 0 to 10 . Given that the observers were not trained anesthesists or surgeons, this score is quite acceptable. Moreover, both observers scored equally well.

Pediatric cardiac nomenclature and outcomes analysis used in this study were derived from an established wellaccepted method, validated by the Society of Thoracic Surgeons (STS) (Jacobs, Jacobs, Maruszewski, Lacour-Gayet, Clarke, Tchervenkov, \& Gaynor, 2005). During the training phase the surgeons wore microphones and two cameras were placed strategically so that observers could also follow the surgical steps. The training cases were taped and stored for later review. The observers created a long hand narrative tool to document the surgical flow from arrival in the OR to patient hand-off in the ICU. Adverse events were classified afterwards by the two observers during the operation as major or minor events, using Catchpole et al.'s (2006).descriptions and examples of minor failure types.

We recorded human factors that include teamwork, fatigue, organizational dynamics, authority gradients, shift work and team performance, using the methods described previously by de Leval and Catchpole (Catchpole, de Leval, McEwan, Pigott, Elliott, McQuillan, \& MacDonald, 2007; de Leval, Carthey, Wright, Farewell, \& Reason, 2000). Three instruments were employed:

1. Mini-STAR: a brief questionnaire that each member of the OR team had to fill in prior to and immediately after each operation. Questions addressed quality of sleep, amount of information received about the patient, possible worries about other team members (all prior to operation), and the occurrence of adverse events and the atmosphere in which the operation was carried out (after operation).

2. Safety culture questionnaire: an extensive questionnaire that had to be filled out twice during the entire study period. Questions were asked about the organizational safety climate (see Bognár, Barach, Johnson, Duncan, Birnbach, Woods, Holl, \& Bacha, 2008).

3. We rated teamwork aspects (four main categories: leadership, situation awareness, decision making and, teamwork and cooperation) on a seven point scale (See Table 2). The scale was used to rate the effect of behavior (shown or absence) on teamwork. In particular, for rating the teamwork aspects, we have drawn upon and slightly modified the NOTECHS (Non-technical skills) system (Flin, Martin, Goeters, Hoermann, Amalberti,Valot, \& Nijhuis, 2003), and the associated ANTS system for rating anaesthetists' non-technical skills (Fletcher, McGeorge, Flin, Glavin, \& Maran, 2003) and the NOTSS system for rating surgeons' nontechnical skills (Yule, Flin, Maran, Paterson-Brown, \& Rowley, 2006a,b). The description of the rating scales themselves was derived from the Observational Teamwork Assessment for Surgery (OTAS ${ }^{\odot}$ ) research instrument (Undre, Healey, Darzi, \& Vincent, 2006). Teamwork aspects were included in a detailed process checklist, consisting of a detailed task analysis of 15 phases involved in the surgical process. Hence, for each subtask, the occurrence of certain activities was recorded, the occurrence of adverse events, as well as the relevant teamwork to deal with these adverse events.

Table 1. Teamwork rating system and rating scale

\begin{tabular}{|l|l|}
\hline \multirow{4}{*}{ Leadership } & Maintenance of standards (MS) \\
\cline { 2 - 2 } & Workload management (WM) \\
\cline { 2 - 2 } & Support of others (SO) \\
\hline Situation Awareness & Notice (SA1) \\
\cline { 2 - 2 } & Understand (SA2) \\
\hline
\end{tabular}


Schraagen $\bullet$ Ven $\bullet$ Barach $\bullet$ Smit

Teams and cardiac surgery

\begin{tabular}{|l|l|}
\hline \multirow{5}{*}{ Decision Making } & Think ahead (SA 3) \\
\hline \multirow{5}{*}{ Teamwork and cooperation } & Definition and diagnosis (DD) \\
\cline { 2 - 2 } & Risk assessment \& option generation (RA) \\
\cline { 2 - 2 } & Outcome review (OR) \\
\cline { 2 - 2 } & Feedback (F) \\
\cline { 2 - 2 } & Backup behaviour (BB) \\
\cline { 2 - 2 } & Coordination (Coor) \\
\hline
\end{tabular}

\begin{tabular}{|l|l|}
\hline 6 & $\begin{array}{l}\text { The shown behaviour was highly effective in enhancing } \\
\text { teamwork }\end{array}$ \\
\hline 5 & $\begin{array}{l}\text { High level of enhancement o f teamwork through shown } \\
\text { behaviour }\end{array}$ \\
\hline 4 & $\begin{array}{l}\text { Moderate enhancement of teamwork through shown } \\
\text { behaviour }\end{array}$ \\
\hline 3 & $\begin{array}{l}\text { Behaviour neither hindered nor enhanced teamwork } \\
\text { needed behaviour }\end{array}$ \\
\hline 1 & $\begin{array}{l}\text { Team work was compromised through lack of certain } \\
\text { needed behaviour }\end{array}$ \\
\hline 0 & The shown behaviour was unclear and hindered teamwork \\
\hline
\end{tabular}

Besides these human factors aspects, we recorded a host of patient variables, such as age, weight, sex, Aristotle Index (a measure for the complexity of the surgery), and number of days of recovery in the ICU, to mention just a few.

\section{RESULTS}

To date, 10 operations have been observed for training purposes, and 15 have been observed for measurement purposes. We have encountered the following challenges in observing and rating the surgical teams:

\section{Challenges in observation}

\section{Complement observations with interviews afterwards}

Observations by themselves are less informative than one might think. They do not provide the reasons behind decisions and actions. It is therefore imperative to complement observations with interviews. This is not always feasible for all team members involved, simply because there are too many team members involved, and because some team members have a busy schedule. Nevertheless, we have found that it works best to make notes of non-routine events during the observation, and to discuss these notes with at least some team members immediately afterwards. For example the time when the surgeon noticed that betadine was spilled on the diathermia patch. The spilling could have compromised the working of the patch, this knowledge was unknown to the observers at that moment.

\section{Exchange of information outside of operating theatre}

We were inclined to limit our observations to the operating theatre and the ICU. It turned out, however, that teams gather and exchange information both before and during the operation outside of the operating theatre. These situations are not taken into account for this study.

\section{Observing technical and non-technical skills during the same operation by one observer}

During our training sessions, it became apparent that our initial optimism regarding the feasibility of observing both technical and non-technical skills at the same time by the same observer had to be tempered. This was aggravated by the fact that we had to observe at least 8 to 9 team members at the same time. Overly focusing on one set of skills necessarily led to a decrement in the accuracy with which the other skills could be observed. We have therefore decided 
to follow a freer format, based on a time line, in which we note an adverse event in one column and hypothesize about the corresponding non-technical skill component in another column. Afterwards, these brief annotations are elaborated. For the same reason, we have deliberately restricted ourselves to observing verbal team-related behavior. Nonverbal behavior, although possibly important and interesting in itself, was not recorded systematically (although the Relational Communication subscale allowed some nonverbal behavior to be recorded).

\section{Act of observing influences the behavior to be observed}

Observers should be aware that, as they become part of the team, and the observations go on for many months, the team will adapt to the observers, will become aware of the hypotheses, and will adapt its behavior accordingly (as far as possible). Of course, there is a limit as to what a team can do. We have not encountered full debriefs and subsequent process remediations, so there will probably be room for improvement after the planned intervention. However, as team members become aware of the necessity of clear communication, for instance, they will speak up, be more alert and attentive and be less ambiguous. An example is the (re)discovery of the 'soap' dispenser in the OR to disinfect the hands. Before the observers joined the team, team members mainly used the 'soap' dispenser outside the OR to disinfect their hands. As a result of observing they started to use the soap dispenser in the OR. Besides this, the team also shows signs of getting used to the observers, like joking with the observers and including them in relaxed talks before and during the surgery. This adds another challenge to the list for the observers, not becoming too involved with the individual people on the OR team. Observers should act as 'fly on the wall' rather than become part of the team, as this may compromise their neutrality. For this reason, too, we have chosen to use human factors experts as observers instead of medical personnel trained in human factors. We have encountered instances where the observers were asked to perform particular tasks within the operational setting. It is much more difficult for a medical person to refuse to cooperate than for a human factors specialist without medical training.

Use observable behavior instead of intentions, thoughts etc.

There is a very natural tendency among observers to attribute particular thoughts and intentions to team members. With infants, injection of cannulas is a very difficult task, because the veins and arteries are hardly visible. This usually increases the preparation time. Especially when it has taken too much time it may occur that two people are working on the baby with syringes. This might be taken as bad workload management or bad planning, however it is actually good behavior of the second person stepping in, as it reduces the stress and increases the success rate. Unless verified afterwards by interviews, this tendency to attribute thoughts or intentions to others needs to be kept in guard. When possible, observers discuss these events with the team member afterwards, and add the explanation to the observations.

\section{Take context into account when assessing non-technical skills}

Communication always take place within a particular context, of which the observers may not always be aware. This could be a particular culture of humour, in which certain team members speak in an apparently derogatory fashion about other team members. Observers may find this not amusing or even insulting, but the team itself may not mind at all. Another set of contextual cues are the arrangements that have been made outside of the operating theatre. For instance, permission to use a particular size of instrument was given by the assistant surgeon prior to the operation. It turned out that this size was inadequate for the particular type of baby who underwent surgery. The first surgeon was unaware of this prior approval. When during the operation, he was confronted with the undersized instrument, and no subsitute was available, he left the operating theatre, angrily demanding of the scrub nurse to immediately find an appropriate instrument. Without knowledge of the prior approval, nobody could properly assess this situation. Observers should be aware that team behavior always takes place in a broader context of social, organizational, political, and cultural factors. When possible observers discuss these events with the team afterwards, and add the explanation to the observations.

\section{CONCLUSIONS}

Our study is designed to identify human factors that are a threat to the safety of children with heart disease. The purpose is to identify the occurrence and types of human factors in the operating room that can potentially lead to undesirable outcomes (i.e. adverse events, near misses and hospital death). In this paper we listed the challenges that we have come across during the preparation and training of the observers. Most challenges in the observations can be solved using interview techniques to verify observations. Another is keeping the awareness of these challenges high and discuss them often with the observers.

Based on the literature we studied, we have made some changes to our tools and procedure to support our study. To date (February 2009), we have attended approximately 15 operations. The observers will observe another 5 operations, after which a major safety intervention and error reduction strategy will be introduced to the staff. The intervention will consist of detailed briefings of the entire OR staff as to incidence and types of intra operative adverse events, as well as 
observed recovery mechanisms. Afterwards each observer will make another set of observations and a comparison will be made, between the two situations. Full data will be available in 2009.

\section{REFERENCES}

Barach, P. , Johnson, J.K., Ahmad, A., Galvan, C., Bognar, A., Duncan, D., Starr, J.P. \& Bacha, E.A. (2008). A prospective observational study of human factors, adverse events, and patient outcomes in surgery for pediatric cardiac disease. The Journal of Thoracic and Cardiovascular Surgery, 136, 1422-1428

Bognár, A., Barach, P., Johnson, J.K., Duncan, R.C., Birnbach, D., Woods, D., Holl, J.L. \& Bacha, E.A. (2008). Errors and the Burden of Errors: Attitudes, Perceptions, and the Culture of Safety in Pediatric Cardiac Surgical Teams. The Annals of Thoracic Surgery, 85, 1374-1381

Boneva, R.S., Botto, L.D., Moore, C.A., Yang, Q., Correa, A. \& Erickson, J.D. (2001). Mortality associated with congenital heart defects in the United States: trends and racial disparities, 1979-1997. Circulation, 103, 2, 376-81.

Bonini, P., Plebani, M., Ceriotti, F. \& Rubboli, F. (2002). Errors in laboratory medicine. Clin Chem., 48, $691-698$.

Cantrill, J.A. \& Cottrell, W.N. (1997). Accuracy of drug allergy documentation. Am J Health Syst Pharm. 54,1, 627629.

Carthey, J., de Leval M.R. \& Reason, J.T. (2001). The human factor in cardiac surgery: errors and near misses in a high technology medical domain. The Annals of Thoracic Surgery, 72, 300-305.

Catchpole, K.R., Giddings, A.E.B., De Leval, M.R., Peek, G.J., Godden, P.J., Utley, M., Gallivan, S., Hirst, G. \& Dale, T. (2006). Identification of systems failures in successful paediatric cardiac surgery. Ergonomics, 49(5-6), 567-588.

Catchpole, K.R., de Leval, M.R., McEwan, A., Pigott, N., Elliott, M.J., McQuillan, A., MacDonald, C. et al. (2007). Patient handover from surgery to intensive care: using Formula 1 pit-stop and aviation models to improve safety and quality. Paediatr Anaesth., 17, 470-478.

Catchpole, K.R., Giddings, A.E.B., Wilkinson, M., Hirst, G., Dale, T. \& De Leval, M.R. (2007). Improving patient safety by identifying latent failures in successful operations. Surgery, 142 (1), 102-110.

Christian, C.K., Gustafson, M.L., Roth, E.M., Sheridan, T.B., Gandhi, T.K., Dwyer, K., Zinner, M.J. et al. (2006). A prospective study of patient safety in the operating room. Surgery 139, 159-73.

Cowell, H.R. (1998). Wrong-site surgery. J Bone Joint Surg Am., 80, 463.

Dambrosia, R. \& Kilpatrick, J. (2002). Medical errors and wrong-site surgery. Orthopedics, 25, 288.

de Leval, M.R., Carthey, J., Wright, D.J., Farewell, V.T. \& Reason, J.T. (2000). Human factors and cardiac surgery: a multicenter study. The Journal of Thoracic and Cardiovascular Surgery, 119, 661-672.

de Leval M.R. (1997). Human factors and surgical outcomes: a Cartesian dream. Lancet, 349, 723-725.

Donchin, Y., Gopher, D., Olin, M., Badihi, Y., Biesky, M., Sprung, C.L., Pizov, R. et al. (1995). A look into the nature and causes of human errors in the intensive care unit. Crit Care Med., 23, 294-300.

Ducat, C.M., Merry, A.F. \& Webster, C.S. (2000). Attitudes and practices of New Zealand anaesthetists with regard to emergency drugs. Anaesth Intensive Care, 28, 692-697.

Eichhorn, J.H. (1989). Prevention of intraoperative anesthesia accidents and related severe injury through safety monitoring. Anesthesiology, 70, 572-577.

Fletcher, G., McGeorge, P., Flin, R., Glavin, R. \& Maran, N. (2003). Anaesthetists' non-technical skills (ANTS). Evaluation of a behavioural marker system. British Journal of Anaesthesia, 90, 580-588.

Flin, R., Martin, L., Goeters, K., Hoermann, J., Amalberti, R., Valot, C. \& Nijhuis, H. (2003). Development of the NOTECHS (Non-Technical Skills) system for assessing pilots' CRM skills. Human Factors and Aerospace Safety, 3 , 95-117.

Galvan, C., Bacha, E., Mohr, J. \& Barach, P. (2005). Analysis of human factors during complex infant cardiac surgical repairs. Progress in Pediatric Cardiology, 20, 13-20.

Helmreich, R.L. (2000). On error management: lessons from aviation. British Medical Journal, 320, 781-785. 
Jacobs, J.P., Jacobs, M.L., Maruszewski, B., Lacour-Gayet, F.G., Clarke, D.R., Tchervenkov, C.I., Gaynor, J.W. et al. (2005). Current status of the European Association for Cardio-Thoracic Surgery and the Society of Thoracic Surgeons Congenital Heart Surgery Database. The Annals of Thoracic Surgery, 80, 2, 278-283; discussion 83-84.

Jenkins, K.J. (2004). Risk adjustment for congenital heart surgery: the RACHS-1 method. Semin Thorac Cardiovasc Surg Pediatr Card Surg Annu., 7, 180-184.

Jenkins, O.F., Morris, R. \& Simpson, J.M. (1997). Australasian perfusion incident survey. Perfusion, 12, $279-288$.

Keenan, R.L. \& Boyan, C.P. (1985). Cardiac arrest due to anesthesia. A study of incidence and causes. JAMA., 253, 2373-2377.

Kohn, L., Corrigan, J. \& Donaldson, M. (2000). To Err is Human: Building a Safer Health System, Washington DC: National Academy Press.

Lemmo, G., Marrocco-Trischitta, M.M., Manni, R. \& Snider, F. (2002). Renal artery pseudoaneurysm and arteriovenous fistula to the inferior vena cava: a late complication following laparoscopic cholecystectomy. Am Surg., $68,143-145$.

Macarthur, D.C., Nixon, S.J. \& Aitken, R.J. (1998). Avoidable deaths still occur after large bowel surgery. Scottish Audit of Surgical Mortality, Royal College of Surgeons of Edinburgh. Br J Surg., 85, 80-83.

Mejak, B.L., Stammers, A., Rauch, E., Vang, S. \& Viessman, T. (2000). A retrospective study on perfusion incidents and safety devices. Perfusion, 15, 51-61.

Mittman, B.S. (2004). Creating the evidence base for quality improvement collaboratives. Ann Intern Med. 140, 897901.

Mohr, J., Batalden, P. \& Barach, P. (2004). Integrating patient safety into the clinical microsystem. Qual Saf Health Care, 13, Suppl 2:ii 34-8.

Murphy, J.F. (2001). The Bristol Royal Infirmary Inquiry 18th July 2001. Ir Med J. 94, 228.

Rao, S., Badani, K.M., Jamieson, K. \& Schildhauer, T. (1996). Pitfalls in the surgical management of cervical spine injuries. Eur Spine J., 5, 153-160.

Reason, J. (1990). Human error, Cambridge, Cambridge University Press.

Shapiro, M.J., Croskerry, P. \& Fisher, S. (2002). Profiles in patient safety: sidedness error. Acad Emerg Med., 9, 326329.

Undre, S., Healey, A., Darzi, A. \& Vincent, C. (2006). Observational assessment of teamwork: A feasibility study. World Journal of Surgery, 30, 1774-1783.

Weick, K.E. \& Quinn, R.E. (1999). Organizational change and development. Annu Rev Psychol., 50, 361-386.

Williamson, L.M., Lowe, S., Love, E.M., Cohen, H., Soldan, K., McClelland, D.B., Skacel, P. et al. (1999). Serious hazards of transfusion (SHOT) initiative: analysis of the first two annual reports. British Medical Journal, 319, 1619.

Wolfson, K.A., Seeger, L.L., Kadell, B.M. \& Eckardt, J.J. (2000). Imaging of surgical paraphernalia: what belongs in the patient and what does not. Radiographics, 20, 1665-1673.

Yule, S., Flin, R., Maran, N., Paterson-Brown, S. \& Rowley, D. (2006a). Non-technical skills for surgeons in the operating room: A review of the literature. Surgery, 139, 140-149.

Yule, S., Flin, R., Maran, N., Paterson-Brown, S. \& Rowley, D. (2006b). Development of a rating system for surgeons' non-technical skills. Medical Education, 40, 1098-1104. 